\title{
BMJ Open The Surveillance After Extremity Tumor Surgery (SAFETY) trial: protocol for a pilot study to determine the feasibility of a multi-centre randomised controlled trial
}

The SAFETY Investigators ${ }^{1,2}$

To cite: The Surveillance After Extremity Tumor Surgery (SAFETY) trial: protocol for a pilot study to determine the feasibility of a multi-centre randomised controlled trial. BMJ Open 2019;9:e029054. doi:10.1136/ bmjopen-2019-029054

- Prepublication history for this paper is available online. To view these files, please visit the journal online (http://dx.doi.org/ 10.1136bmjopen-2019-029054)

Received 12 January 2019

Revised 22 August 2019

Accepted 30 August 2019

\section{Check for updates}

(C) Author(s) (or their employer(s)) 2019. Re-use permitted under CC BY-NC. No commercial re-use. See rights and permissions. Published by BMJ.

${ }^{1}$ Department of Surgery, McMaster University, Hamilton Ontario, Canada

2Juravinski Cancer Centre, Hamilton Health Sciences, Hamilton, Ontario, Canada

Correspondence to The SAFETY Investigators; mghert@hhsc.ca

\section{ABSTRACT}

Introduction Following the treatment of patients with soft tissue sarcomas (STS) that are not metastatic at presentation, the high risk for local and systemic disease recurrence necessitates post-treatment surveillance. Systemic recurrence is most often detected in the lungs. The most appropriate surveillance frequency and modality remain unknown and, as such, clinical practice is highly varied. We plan to assess the feasibility of conducting a multi-centre randomised controlled trial (RCT) that will evaluate the effect on overall 5-year survival of two different surveillance frequencies and imaging modalities in patients with STS who undergo surgical excision with curative intent.

Methods and analysis The Surveillance After Extremity Tumor Surgery trial will be a multi-centre $2 \times 2$ factorial RCT. Patients with non-metastatic primary Grade II or III STS treated with excision will be allocated to one of four treatment arms ${ }^{1}$ : chest radiograph (CXR) every 3 months for 2 years ${ }^{2}$; CXR every 6 months for 2 years ${ }^{3}$; chest CT every 3 months for 2 years or ${ }^{4}$ chest CT every 6 months for 2 years. The primary outcome of the pilot study is the feasibility of a definitive RCT based on a combination of feasibility endpoints. Secondary outcomes for the pilot study include the primary outcome of the definitive trial (overall survival), patient-reported outcomes on anxiety, satisfaction and quality of life, local recurrence-free survival, metastasis-free survival, treatment-related complications and net healthcare costs related to surveillance.

Ethics and dissemination This trial received provisional ethics approval from the McMaster/Hamilton Health Sciences Research Ethics Board on 7 August 2019 (Project number 7562). Final ethics approval will be obtained prior to commencing patient recruitment. Once feasibility has been established and the definitive protocol is finalised, the study will transition to the definitive study. Trial registration NCT03944798; Pre-results.

\section{BACKGROUND}

Magnitude of the problem

Sarcomas are malignancies of connective tissue that most commonly occur in the extremities. Sarcomas can arise within bone
Strengths and limitations of this study

- The Surveillance After Extremity Tumor Surgery (SAFETY) trial will be an international multi-centre $2 \times 2$ factorial randomised controlled trial.

- The trial will answer a high priority question for sarcoma surgeons.

- The SAFETY trial will build on the international collaboration and experience of the Prophylactic Antibiotic Regimens in Tumor Surgery (PARITY) trial.

- The feasibility pilot study is essential before undertaking this large multi-centre trial.

- The success of the pilot study is dependent on the ability of clinical sites to recruit patients, comply with the protocol and complete high-quality follow-up data.

(bone sarcoma) or soft tissue (soft tissue sarcoma (STS)). Chemotherapy is not curative for the vast majority of patients with $\mathrm{STS}^{1}$; therefore, surgery is the standard treatment for STS, with radiation considered important for local disease control.

Following treatment for a STS that is not metastatic at presentation, the risk for local and systemic disease recurrence necessitates careful postoperative surveillance. Between $40 \%$ and $50 \%$ of all patients with sarcoma will develop a local or distant recurrence; however, the risk of recurrence is greatest in the first few years, with $68 \%$ occurring by 2 years and $90 \%$ by 5 years. ${ }^{2-4}$ Metastasis to the lung is the most frequent single location of disease recurrence in patients with sarcoma, occurring in the majority of patients with metastases. ${ }^{4-7}$ Therefore, routine follow-up after completing sarcoma treatment is standard practice in the first 5 years after surgery. These visits typically include a clinical history, physical examination and imaging 
of the lungs (chest radiograph (CXR) or CT scan of the lungs).

Surveillance strategies for long-term follow-up of patients with sarcoma have not been well researched and current guidelines are based on expert opinion, not on high-quality evidence. ${ }^{89}$ As such, current clinical practice is highly varied, with survey data of musculoskeletal oncologists showing that the number of clinic visits ranges from 2 to 12, the number of CXRs obtained ranges from 0 to 13 , and the number of CT scans ranges from 1 to 8 in the first year of surveillance. ${ }^{10-12}$ The current National Comprehensive Cancer Network (NCCN) guidelines suggest that stage II or III tumours should be followed with chest imaging (CT or CXR) every 2-6 months for the first 2-3years and then annually thereafter, while stage I tumours could be followed less frequently during the first 2-3 years. ${ }^{13}$

\section{Best evidence for surveillance strategies}

Post-treatment STS surveillance is an integral element of patient care. Although earlier detection of metastatic disease may improve long-term survival, no study has yet provided definitive evidence to support this assumption. In order to assess the available evidence, we completed a systematic review of the available randomised controlled trial (RCT) evidence for surveillance in sarcoma management. ${ }^{14}$ A single study (published separately with early and longer-term follow-up) was identified. ${ }^{15} 16$ The authors of this single-centre study found that 3-year overall and disease-free survival was not worse in patients with sarcoma who had less intensive surveillance (CXRs) than those with more intensive surveillance (CT scans) ${ }^{15}$ Due to the sample size, this trial could not conclusively demonstrate non-inferiority in overall or diseasefree survival for a 6-month interval of follow-up visits against 3-month interval (both were 64\% and 69\%, respectively). ${ }^{15}$

A follow-up study on the same patient cohort with 5-year survival outcomes confirmed that more frequent follow-up did not improve survival and that, although CT scans detected pulmonary metastasis earlier, they did not lead to better survival compared with CXRs. ${ }^{16}$ However, this was a single-centre study with relatively small numbers and, therefore, confidence in the results and generalisability of the data to other centres is limited. In addition, a relatively small proportion of screened patients (42\%) that were eligible for the trial were included due to the exclusion of patients unlikely to follow-up, thus possibly introducing selection bias. ${ }^{15}$ Furthermore, low-grade sarcomas were eligible and included in this study, even though they have little metastatic potential and tumour-related mortality; their inclusion may have diminished the magnitude of the effects of the interventions. ${ }^{15}$ Finally, the majority of the included patients were patients with bone sarcoma, thereby limiting the interpretation to patients with STS. ${ }^{15}$

\section{Risks and benefits of intensive surveillance}

Regular, intensive surveillance is more likely to identify recurrent disease earlier than would less intensive surveillance. This type of surveillance may provide reassurance to patients and clinicians; however, the adverse effects of intensive surveillance practices are also noteworthy. The costs that healthcare systems incur as a result of sarcoma surveillance are substantial and could be in excess of US\$20000 for high-grade sarcomas. ${ }^{17}$ Furthermore, intensive surveillance can threaten the financial security of patients, due in part to the direct (including travel, accommodation, personal care and homemaking) and indirect costs (including lost wages for patients and their caregivers) incurred as a result of follow-up appointments. ${ }^{18}$ As a result, patients' health and quality of life can be dramatically impacted. ${ }^{18-20}$

Secondary investigations and earlier knowledge of disease recurrence can also induce anxiety and impact the psychosocial well-being for those whose mortality risk cannot be significantly reduced by further medical interventions. ${ }^{21}$ Overcrowded clinics and long wait times may constitute other important factors that affect patients' psychosocial well-being. ${ }^{22}$ Finally, the use of CT has raised concerns over unnecessary radiation exposure compared with radiographs, although lower-dose CT scans may mitigate some of these concerns. ${ }^{23}$

\section{Surveillance research as a priority in orthopaedic oncology}

We recently published a modified Delphi study in which we aimed to identify a clinically relevant consensus-based research agenda in the sarcoma field. ${ }^{24}$ From this Delphi process that included 80 orthopaedic oncologists and patient representation (with participation from 18 countries), we identified critical research priorities in the field of orthopaedic oncology and determined the top four feasible and important research questions that will directly inform patient care and enhance clinical practice. This study identified the evaluation of postoperative surveillance strategies as the highest-ranking research priority in the sarcoma field. ${ }^{24}$

\section{PATIENT AND PUBLIC INVOLVEMENT}

To ensure that we maintain a patient-centred approach to the design and development of this study, we required the opportunity for open dialogue between the multidisciplinary and international Surveillance After Extremity Tumor Surgery (SAFETY) study team, along with patient/caregiver representatives and other key stakeholders. To facilitate their interaction and collaboration, we held an in-person Protocol Development Meeting in Toronto, ON, Canada in May 2018. At this meeting, we made critical decisions with respect to the study protocol, including (A) study design; (B) primary and secondary outcomes; (C) patient eligibility; (D) follow-up time frame; (E) methods to protect against bias; (F) randomisation stratification and $(G)$ further patient engagement. We also had the opportunity to discuss several issues that 
$\mathrm{M}=$ month; $\mathrm{CXR}=$ chest $\mathrm{X}$-ray; $\mathrm{CT}=$ computed tomography

Figure 1 Study of flow diagram.

may compromise the study's success and strategise ways to manage these challenges, such as (i) acceptable surveillance schedules that account for differences in international standards of clinical practice; (ii) possible ethical concerns; (iii) patient compliance; (iv) local implementation and procedural variation; (v) competing studies and (vi) funding opportunities.

We are also conducting a patient survey to assess international patient willingness to participate in a study that randomises patients to a postoperative surveillance regimen in the management of a primary extremity sarcoma. Since there is no available validated tool to assess patient opinions and preferences, we developed a unique patient questionnaire for the purposes of this

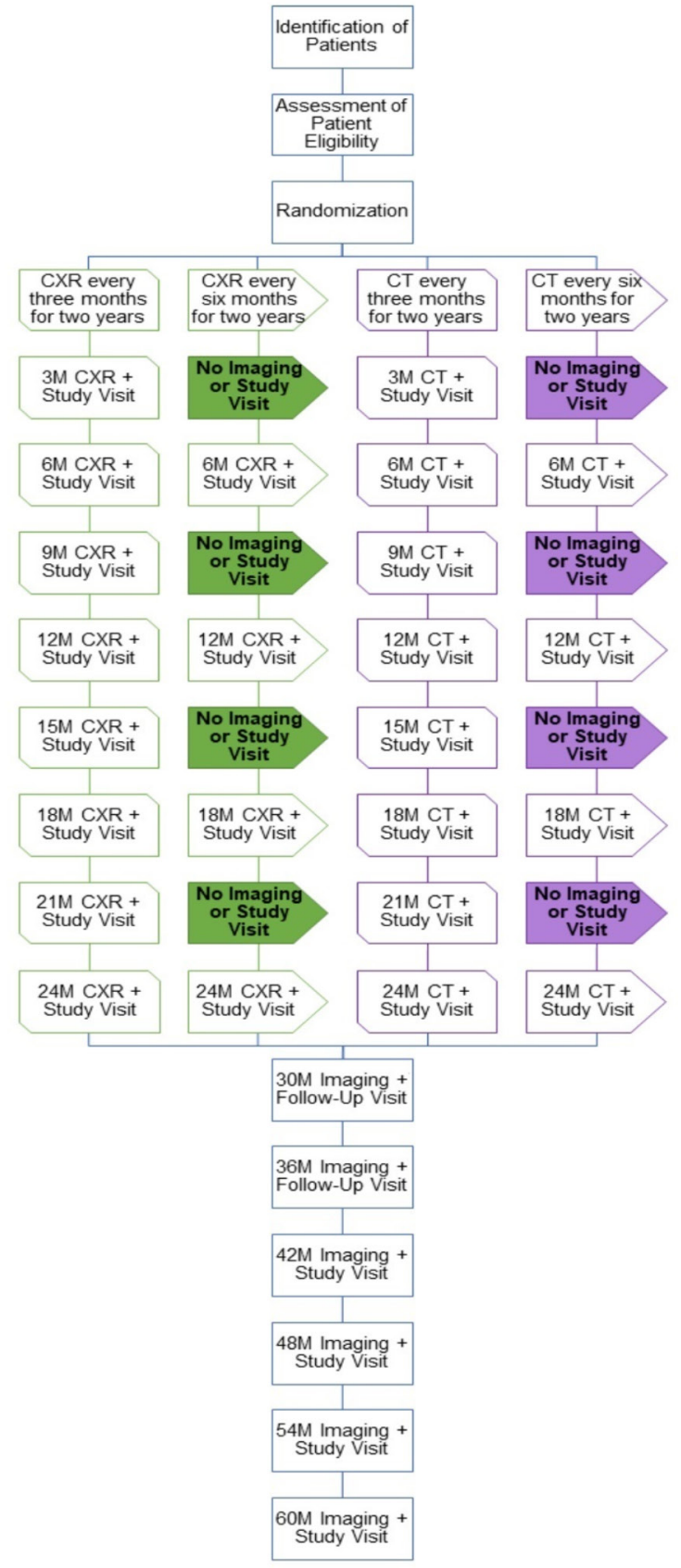

study. All new patients who present to a participating sarcoma clinic are screened for study participation. The preliminary survey questionnaire responses suggest that most patients with sarcoma believe that they have a good understanding of clinical research. Furthermore, over half of respondents feel comfortable with being randomised to receive a treatment. Ultimately, almost $80 \%$ of respondents have indicated that they would agree to participate in the SAFETY trial if eligible.

\section{STUDY DESIGN}

We plan to assess the feasibility of conducting the pragmatic, international, multi-centre, $2 \times 2$ factorial SAFETY 
RCT that answers the following questions: In extremity, patients with STS who undergo surgical resection with curative intent, ${ }^{1}$ what is the impact of surveillance frequency (every 3 vs every 6 months) on overall survival at 5 years, and ${ }^{2}$ what is the impact of surveillance imaging modality (CXR vs CT scan) on overall survival at 5 years? To assess feasibility, we will conduct a pilot study. Study participants will be randomised to one of four possible treatment arms (see Study Interventions later). Randomisation will occur at the end of active treatment (surgery \pm systemic treatment \pm local radiation). Following the 2-year intervention phase, study participants will continue to be assessed at regular intervals for an additional 3 years. As such, all pilot study patients will be transitioned into the definitive study and be included in it. Details of the flow of each study arm are outlined in figure 1 . We anticipate the duration of the pilot study to be 3 years in order to collect intervention phase data on all participants. The primary outcome of the pilot study is the feasibility of a definitive RCT based on a combination of feasibility endpoints.

The $2 \times 2$ factorial study design is ideal and the most efficient method to study two treatment interventions in a single RCT, particularly when there is no interaction between the two interventions. This is unlike a scenario in which the two interventions are medications that may have a synergistic or negative effect when combined. A Bayesian design would be useful do avoid the question of whether or not an interaction exists, however for the purposes of the present trial it is clear that no interaction exists between the frequency and intensity of surveillance. As Freidlin and Korn discuss in their commentary, the $2 \times 2$ factorial design is an efficient design to evaluate two interventions in a cancer clinical trial when there are no interactions between treatments. ${ }^{25}$

\section{OBJECTIVES}

\section{Pilot study primary research objectives}

The primary objective of the pilot study will be to determine whether it is feasible to conduct a large multi-centre RCT that will evaluate the impact of surveillance strategies on patient survival following extremity STS surgery. To do so, we will assess our ability to:

1. Recruit patients across multiple participating clinical sites;

2. Ensure compliance with the study protocol, including the application of eligibility criteria, timing of intervention phase and post-intervention phase visits and imaging modality;

3. Maintain completeness of follow-up data;

4. Maintain completeness of cost analysis data and

5. Maintain data quality.

\section{Pilot study secondary research objectives}

The secondary objectives of the pilot study will include assessing the impact of either surveillance frequency (every 3 vs every 6 months) or imaging modality (CXR vs CT scan) on:

1. Overall survival;

2. Patient anxiety, satisfaction and quality of life;

3. Local recurrence-free survival (LRFS) and metastasis-free survival (MFS);

4. Treatment-related complications and

5. Net direct healthcare costs and net costs of treatment and treatment-related complications once metastases are detected.

\section{HYPOTHESIS}

\section{Pilot study}

We hypothesise that the SAFETY trial will be feasible due to (A) its pragmatic design; (B) our established international collaborative research network; (C) our qualified, multidisciplinary study team; (D) our existing trial infrastructure and (E) the priority of the study question.

\section{Definitive study}

There are two hypotheses:

1. More frequent postoperative surveillance (compared with less frequent postoperative surveillance) in the first 2 years following the surgical excision of a STS will improve survival over 5 years;

2. The use of postoperative CT scans (compared with CXR) in the first 2 years following the surgical excision of a STS will improve survival over 5 years.

\section{STUDY SETTING}

This study will be coordinated by the Methods Centre within the Centre for Evidence-Based Orthopaedics at McMaster University (Hamilton, ON, Canada). For the pilot study, we expect that patients will be enrolled from 10 clinical sites across four continents. Clinical sites will be carefully screened prior to participation in the study. The clinical site inclusion criteria are as follows: (i) adequate research personnel and infrastructure to manage the study; (ii) sufficiently high extremity STS volume to complete enrolment within the study timeline (defined as greater than or equal to $(\geq) 20$ patients per year); (iii) commitment from all or most orthopaedic oncologists to participate in the trial and (iv) access to the two imaging modalities. The exclusion criteria are as follows: (i) a lack of interest in the trial; (ii) anticipated challenges with protocol compliance; (iii) conflicting studies, in the judgement of the Principal Investigator, that would inhibit patient participation and (iv) financial or contract constraints.

\section{PATIENT ELIGIBILITY CRITERIA Inclusion criteria}

Patients who meet all of the following criteria will be included:

1. Age of 18 years or older; 
2. Diagnosed with a primary extremity grade II or III STS;

3 . Undergone surgical resection of the tumour with curative intent and grossly negative margins (R0 or R1 resection margins);

4. Completed neoadjuvant or adjuvant radiation and/or chemotherapy, if applicable;

5 . The tumour size is greater than or equal to $(\geq) 5 \mathrm{~cm}$ according to the pathology report or pre-treatment MRI if neoadjuvant radiation and/or chemotherapy are given; and

6. Provision of informed consent.

\section{Exclusion criteria}

Patients who meet any of the following criteria will be excluded:

1. Metastatic disease at initial presentation based on thoracic imaging (a second CT scan may be required to confirm that indeterminate nodules are false positives before the patient can be enrolled provided that the second CT scan shows no evidence of metastatic disease);

2. Undergone surgical excision of a local recurrence;

3. Diagnosis of one of the special subtypes: myxoid/ round cell liposarcoma or extra-skeletal Ewing's sarcoma (these sarcomas have different metastatic patterns, which necessitate different surveillance protocols);

4. Previous diagnosis of a genetic syndrome with an elevated risk of malignancy, such as Li-Fraumeni Syndrome (such individuals appear to be at an elevated risk for radiation-induced cancers, so the use of CT scans should be limited);

5. Previous diagnosis with a comorbid condition that has a life expectancy of less than 1 year;

6 . The site-specific surveillance protocol for the patient's disease is not compatible with the study protocol (ie, regular planned whole-body imaging with positron emission tomography scans);

7. Diagnosed with another malignancy within the past 5 years;

8. Likely problems, in the judgement of the investigator, with maintaining follow-up;

9. Currently enrolled in a study that does not permit co-enrollment;

10. The patient has already been enrolled in the SAFETY trial.

\section{RECRUITMENT AND SCREENING}

Each participating clinical site will have a locally responsible investigator who will oversee the local administration of the trial, screen patients with STS for eligibility and develop a site-specific patient enrolment plan. A screening form will be completed for all patients with STS aged 18 years or older, irrespective of whether they are eligible to participate in the study or not. Patients will become eligible, will be screened and consented during the first clinic visit at which all treatment is complete, the surgical wound has healed, and the plan for post-treatment surveillance is discussed with the patient. The process of obtaining and documenting informed consent will be completed in accordance with local Good Clinical Practice recommendations. Consent procedures will comply with the appropriate ethics committee and the Health Insurance Portability and Accountability Act (where applicable).

\section{RANDOMISATION AND ALLOCATION OF PATIENTS TO STUDY GROUPS}

A centralised and automated internet-based randomisation system using random variable block sizes will assign participants to the study groups. Study personnel at each participating site will complete this task. Randomisation will occur only after eligibility is confirmed and consent to participate has been obtained. Participants will be stratified based on clinical site and perioperative chemotherapy.

\section{STUDY INTERVENTIONS}

Participants will be randomised to one of four treatment groups:

1. CXR every 3 months for 2 years;

2. CXR every 6 months for 2 years;

3. Chest CT every 3 months for 2 years or

4. Chest CT every 6 months for 2 years.

Following completion of the intervention phase, participants will continue to be followed in the study for an additional 3 years. During this 3-year post-intervention phase, participants will be followed at least every 6 months as per NCCN guidelines. ${ }^{13}$ If possible, thoracic imaging will continue at each scheduled post-intervention phase visit according to the participants' original allocations.

\section{RELAPSE}

Local imaging and clinical assessment of the primary tumour site will be carried out as per the standard protocol at each participating clinical site. Further diagnostic tests will be performed in the presence of clinical symptoms or radiologic findings suggestive of disease relapse. Recurrence will be radiologically or histologically confirmed and classified as local or systemic (metastasis) recurrence. The first modality suggesting disease relapse in participants with confirmed local or systemic recurrence will be recorded as responsible for its detection.

\section{OUTCOME MEASURES}

\section{Pilot study primary outcome}

To evaluate feasibility, we will assess the number of patients screened and recruited at each participating clinical site, participant retention and maintenance of data quality. In addition, we will evaluate the utilisation of an internet-based centralised randomisation system focusing 
on the accuracy of data entry, appropriate stratification of participants and the minimisation of randomisation errors. Finally, we will evaluate investigator and participant compliance with the study protocol, including the application of eligibility criteria, compliance with the surveillance imaging and frequency regimens, frequency of crossover and timing of post-intervention phase visits. As discussed by Moore $e t a l$, the pilot study will investigate the process of the proposed definitive trial rather than its outcomes. ${ }^{26}$ The a priori criteria for the success of the pilot study are listed below:

1. Recruitment measure: We will consider our recruitment strategy feasible if we are able to enrol the pilot sample of 195 patients (approximately 20 patients from each clinical site participating in the pilot study) within 2 years. See sample size determination later. As such, we will aim to recruit 100 patients during the first year. If we are unable to achieve at least $90 \%$ of this goal (90 patients), then we will plan to increase the number of participating sites as a study rescue measure.

2. Protocol adherence measure: During the pilot study of the PARITY trial, we were able to maintain an overall protocol adherence rate in excess of $90 \% .{ }^{27}$ Recent reports prepared for the PARITY Data and Safety Monitoring Board (DSMB) indicate a similar protocol adherence rate. However, given the greater complexity and longer duration of the SAFETY trial interventions, we will consider our protocol adherence strategies feasible if there is adherence of $85 \%$ or greater to the visit windows and imaging modality prescribed by the protocol.

3. Participant retention measure: While $20 \%$ loss-to-follow-up has traditionally been considered acceptable in clinical research, evidence from other orthopaedic trials suggests that bias begins to affect study results at even lower rates of loss-to-follow-up. ${ }^{28}$ Therefore, we will consider our participant retention strategies feasible if no more than $15 \%$ of participants are lost-to-follow-up.

4. Maintenance of data quality measure: We obtained a data completeness rate of approximately $90 \%$ in the PARITY trial pilot study. ${ }^{27}$ Therefore, we will consider our data quality strategies feasible if we are able to maintain $95 \%$ or greater completeness of participant follow-up data for the definitive primary outcome. We will also consider our data quality strategies feasible if we are able to maintain $85 \%$ or greater completeness of participant follow-up data for the secondary outcomes.

\section{Pilot study secondary outcomes}

Death from any cause will be recorded during the pilot study. Data on secondary outcomes for the definitive trial, which are listed below, will also be collected. These include:

1. Patient-reported outcome measures: The validated PatientReported Outcomes Measurement Information System (PROMIS) Cancer-Anxiety questionnaire, PROMIS Satisfaction with Social Roles and Activities questionnaire and the EuroQol-5 Dimension will be used to assess patient anxiety, satisfaction and quality of life, respectively.
These questionnaires will be administered at the baseline visit, as well as the 6-month, 12-month, 18-month and 24-month intervention phase, as well as 36-month, 48-month and 60-month post-intervention phase visits.

2. LRFS outcome measure: LRFS will be defined as the length of time from randomisation that the participant survives with no detection of recurrent disease at the initial tumour site or operative field.

3. MFS outcome measure: MFS will be defined as the length of time from randomisation that the participant survives with no detection of systemic disease recurrence at any anatomic location.

4. Treatment-related complications outcome measures: Treatment-related complications will include both chemotherapy-related complications, such as febrile neutropaenia, fungal infections or sepsis and thoracotomy-related complications, such as pneumothorax or surgical site infections.

5. Net healthcare costs outcome measures: We will perform an incremental cost analysis of net costs of surveillance and costs incurred from metastasis treatment and metastasis treatment-related complications. Unit costs for all resources used by trial participants will be obtained from regional statistics and from centres participating in the trial. These unit costs will be combined with the resource volumes to obtain a net cost per participant over their time in the trial.

\section{PROTECTING AGAINST SOURCES OF BIAS Adjudication of outcomes}

An independent Central Adjudication Committee (CAC) will review all situations where eligibility is in doubt, as well as all reported instances of disease relapse, treatment-related complications and death to determine whether a study event has occurred. The SAFETY CAC will be comprised of two orthopaedic oncologists, one medical oncologist and one radiologist. All participating clinical sites will submit digital imaging and relevant hospital records to the Methods Centre via a web-based platform for events that require adjudication.

\section{Blinding}

The local clinical team, site study personnel and participants cannot be blinded to the treatment allocation. The CAC will be blinded to surveillance frequency. The data analysts will, however, remain blinded during the trial's analysis.

\section{Maximisation of follow-up}

We anticipate only minimal losses to follow-up in our musculoskeletal oncology population. Nonetheless, the following procedures will be implemented to minimise losses:

- Individuals likely to present problems with compliance to the study protocol or maintaining follow-up will be excluded;

- At the time of randomisation, participants will be asked to provide their contact information, as well as the 
contact information of their family physician and three alternate contacts;

- Participants who refuse to return for a study assessment will be asked if they are willing to provide follow-up data (to determine survival and to complete study questionnaires) via telephone;

- If a participant cannot be reached, their status regarding the primary study outcome will be assessed by reviewing their medical records;

- Study personnel will remind participants of upcoming clinic visits;

- To assuage possible concerns related to less frequent follow-up, participants will be encouraged to schedule an ad hoc visit anytime they are concerned, even if it breaks the surveillance protocol to which they were assigned;

- Participants will be provided with access to educational content, such as a video that demonstrates how to self-examine for a local recurrence of their STS and

- Parking and travel vouchers will be provided to participants, where possible, to alleviate the costs associated with the study.

\section{Minimisation of crossovers of surveillance interventions}

Crossovers are unlikely for either surveillance intervention as investigators will be requesting the imaging modality during surveillance visits. Any deviation with regards to frequency or imaging modality will be documented. In the event of disease recurrence or progression, the following standardised management protocols will be adopted:

- Local recurrence: the participant will have a lung CT scan to confirm no progression of their systemic disease before continuing with the study protocol.

- Metastases: the participant will no longer be followed as per the study protocol, but per the appropriate follow-up for the interventions required for the treatment of metastases; however, the participant will continue to be followed in the trial.

For both events, the specific imaging modality used to detect either the local recurrence or the metastases will be documented.

Patients that have incidental or off-protocol imaging will not crossover; however, this will be documented as a protocol deviation. In the case of a CXR that warrants further investigation with a CT scan, this will be documented. If the patient is found to have disease recurrence, we will document how the disease recurrence was (A) first identified and (B) confirmed. If after a CT scan the patient is found to not have disease recurrence, the patient will resume surveillance as per the arm to which they were randomised.

\section{SAMPLE SIZE DETERMINATION}

\section{Pilot study sample size}

The CI approach was used to calculate the required sample size for the pilot study. ${ }^{29}$ We determined a priori that the definitive trial would only be feasible if our
Table 1 Sample size per group for $80 \%$ power, $\alpha=0.05$

\begin{tabular}{lrrrrr}
\hline & \multicolumn{5}{c}{$\begin{array}{c}\text { Event rate in more intensive } \\
\text { surveillance group }\end{array}$} \\
\cline { 2 - 6 } & & $\mathbf{2 5 \%}$ & $\mathbf{3 0 \%}$ & $\mathbf{3 5 \%}$ & $\mathbf{4 0 \%}$ \\
\hline Event rate in less & $35 \%$ & 696 & 2832 & \multicolumn{1}{c}{-} & - \\
intensive surveillance & $40 \%$ & 332 & 752 & 3020 & - \\
group & $45 \%$ & 196 & 352 & 792 & 3148 \\
& $50 \%$ & 132 & 204 & 368 & 816 \\
& $55 \%$ & 96 & 136 & 212 & 372 \\
\hline
\end{tabular}

Event rate=death

protocol adherence rate was at least $85 \%$. Using a $95 \%$ $\mathrm{CI}$ and a $5 \%$ margin of error, we calculated a required sample size of 195 patients.

\section{Definitive study sample size}

Our best estimate of the control group overall 5-year survival for both the surveillance frequency and imaging modality is $55 \% .{ }^{16}$ Given that intensive surveillance will detect metastatic disease at an earlier stage, we will use a superiority design to compare survival between more versus less intensive surveillance. A $10 \%$ absolute increase in overall 5-year survival associated with both more frequent surveillance and the use of CT scans represents a clinically important difference, as outlined by the American Society of Clinical Oncology's statement on clinically meaningful outcomes in cancer trials. ${ }^{30}$ Therefore, the definitive trial will be powered to detect an absolute difference of $10 \%$ in overall 5 -year survival.

With a desired power of 0.80 , we calculated a sample size of 396 participants per study arm. We will account for a $5 \%$ loss to follow-up and, therefore, the final sample size will be 830 participants. Table 1 shows various sample sizes for pairwise comparisons of alternative surveillance frequencies/imaging modalities given varying control event rates and absolute increases in survival. Statistical Package for the Social Sciences (IBM Corporation) software was used for sample size calculation.

The definitive sample size calculation may be adjusted as we prepare for the transition from the pilot to the definitive study as a result of data collected during the pilot study. One factor we may consider will be the percent lost to follow-up by the end of the pilot study. Other factors such as the estimated control group overall 5-year survival, the clinically meaningful outcome and power cannot be amended. The rationale for transition of subject data from the pilot study to the definitive study has previously been discussed. ${ }^{31}$ It is acceptable to pool the data if the study methods are not adjusted following the pilot study, and the research tools are standardised.

\section{ANALYSIS OF FEASIBILITY OUTCOMES}

A full description of the measures, variables and methods of analysis are shown in table 2 . We will record 
Table 2 Summary of feasibility outcomes analysis plan

\begin{tabular}{|c|c|c|c|}
\hline Objective & Outcome & $\begin{array}{l}\text { Criteria for success of } \\
\text { feasibility }\end{array}$ & Method of analysis \\
\hline \multirow{4}{*}{$\begin{array}{l}\text { To determine the feasibility of } \\
\text { conducting the multi-centre } \\
\text { SAFETY international RCT }\end{array}$} & Recruitment measure & $\begin{array}{l}\text { Enrolment of pilot sample } \\
\text { within } 2 \text { years }\end{array}$ & \multirow{4}{*}{$\begin{array}{l}\text { Descriptive statistics- } \\
\text { reported as counts (percent) } \\
\text { for categorical variables and } \\
\text { means (SD) for continuous } \\
\text { variables with } 95 \% \mathrm{Cl}\end{array}$} \\
\hline & Protocol adherence measure & $\begin{array}{l}\text { Protocol adherence of } 85 \% \text { or } \\
\text { greater }\end{array}$ & \\
\hline & Participant retention measure & $\begin{array}{l}\text { Loss-to-participant follow-up } \\
\text { of } 15 \% \text { or less }\end{array}$ & \\
\hline & $\begin{array}{l}\text { Maintenance of data quality } \\
\text { measure }\end{array}$ & $\begin{array}{l}\text { Data completeness of } 95 \% \\
\text { or greater for the definitive } \\
\text { primary outcome } \\
\text { Data completeness of } 85 \% \\
\text { or greater for the secondary } \\
\text { outcomes }\end{array}$ & \\
\hline
\end{tabular}

$\mathrm{RCT}$, randomised controlled trial; SAFETY, Surveillance After Extremity Tumor Surgery.

the total number of participants enrolled on a monthly basis. Each participating site will keep a Screening Log of included and excluded patients. We will also keep a record of participants who miss visits, and those who are withdrawn or lost to follow-up. These will be reported using descriptive statistics-reported as counts (percent) for categorical variables and mean (SD) for continuous variables with $95 \%$ CIs. We will report the proportion of complete Case Report Form (CRFs) as descriptive data.

\section{ANALYSIS OF DEFINITIVE STUDY PRIMARY OUTCOME}

The analysis and reporting of the trial will follow the Consolidated Standards of Reporting Trials criteria. ${ }^{32}$ The primary analysis will compare the treatment groups on the overall 5-year survival. Two independent comparisons between treatment groups will be made using Cox regression models with time to the definitive primary endpoint. ${ }^{33}$ Results will be expressed as effect (ORs for binary outcomes, HRs for time-dependent outcomes and mean difference for continuous outcomes), corresponding two-sided $95 \%$ CIs and associated p-values.

\section{ETHICAL CONSIDERATIONS}

This study is to be conducted according to international standards of Good Clinical Practice, applicable government regulations and institutional research policies and procedures. All study intervention phase (surveillance) arms fall within the spectrum of current standard practice, as do the standardised post-intervention phase follow-up visits. The study protocol will be submitted to a properly constituted independent ethics committee, in agreement with local legal prescriptions, for formal approval of the study conduct at each participating clinical site. A copy of this approval will be provided to the Methods Centre by each participating clinical site prior to the local commencement of the study.

\section{STUDY TIMELINE}

We expect that the pilot study will take just over 3 years to complete. We estimate that recruitment will take approximately 1 year to complete per site. The initiation of screening and enrolment will likely be staggered across the participating clinical sites due to the variability in the time required to obtain ethics approval and negotiate institutional contracts. Therefore, the pilot study recruitment timeline will be up to 2 years. We expect a further 1 year for all pilot participants to complete the intervention phase of the trial. Although we will not have complete post-intervention phase data for any pilot participants, we anticipate being able to determine feasibility at the end of the intervention phase based on our feasibility objectives. We plan a priori to transition directly from the pilot to the definitive study if feasibility is established.

\section{DATA SAFETY MONITORING BOARD}

As per the principles established by the Data Monitoring Committees: Lessons, Ethics, Statistics Study Group charter, a DSMB will oversee the safety of the trial participants and the overall conduct of the trial. The Committee members will be independent of the trial, free of conflicts with any of the investigative team, and will consist of two orthopaedic oncologists, a medical oncologist, a radiologist and a biostatistician. The DSMB will frequently review enrolment and demographic summaries, listings of protocol deviations, and summaries and listings of serious adverse events. They will advise the Principal Investigator and SAFETY study team on any concerns related to participant safety and trial conduct and will make recommendations for (A) study continuation as designed; (B) study termination; (C) study continuation with major or minor modifications or (D) temporary study suspension of enrolment until some uncertainty is resolved. 


\section{KNOWLEDGE DISSEMINATION}

The results of the study will be submitted for publication regardless of whether there are significant findings, as well as posted on ClinicalTrials.gov. The trial has been registered on clinicaltrials.gov. In addition to scientific manuscripts and presentations, we plan to prepare study reports and press releases for patients and other stakeholders that are transparent, and that the language is understandable to the general public.

\section{POTENTIAL IMPACT OF THE STUDY}

The benefit of this pilot study would be to determine the feasibility of the SAFETY trial. This is essential prior to undertaking a large multi-centre RCT. Experience gained during the pilot study will provide insight into methods to increase enrolment, strategies to maintain protocol adherence and the adjustment of recruitment expectations. In addition, the ultimate success of the pilot study will support funding requests for the definitive study of the multi-centre SAFETY trial.

Once the feasibility endpoints are reached, we will transition directly into and begin recruiting for the definitive SAFETY trial. The ultimate goal of the SAFETY trial is to provide high-quality evidence for surveillance strategies following the treatment of STS, which will allow for the development of evidence-based clinical practice guidelines for patients with sarcoma worldwide.

Acknowledgements We would like to thank our patient advisers, Nina Szpakowski and Arlene Manherz, for their contributions.

Collaborators The SAFETY Investigators: Michelle Ghert, MD, FRCSC (Steering Committee Chair), Department of Surgery, McMaster University (Hamilton, Ontario, Canada); Mohit Bhandari, MD, PhD, FRCSC, Department of Surgery \& Department of Health Research Methods, Evidence and Impact, McMaster University (Hamilton, Ontario, Canada); Anthony Bozzo, MD, Department of Surgery, McMaster University (Hamilton, Ontario, Canada); PD Sander Dijkstra, MD, PhD, Department of Orthopaedics, Leiden University Medical Center (Leiden, the Netherlands); Anthony Griffin, MSc, Musculoskeletal Oncology Unit, Mount Sinai Hospital (Toronto, Ontario, Canada); Robert Grimer, MB BS, DSc, FRCS, FRCS Ed(Orth), Department of Surgery, University of Birmingham (Birmingham, United Kingdom); James Hayden, MD, PhD, FACS, Department of Orthopaedics \& Rehabilitation, Oregon Health \& Science University (Portland, Oregon, USA); Arlene Manherz, (Community); Karim Masrouha, MD, Department of Surgery, McMaster University (Hamilton, Ontario, Canada); Paula McKay, BSc, Department of Surgery, McMaster University (Hamilton, Ontario, Canada); Benjamin Miller, MD, MS, FACS, Department of Orthopaedics \& Rehabilitation, University of lowa (lowa City, lowa, USA); Naveen Parasu, MD, MRCP (UK), MRCR (UK), FRCPC, Department of Radiology, McMaster University (Hamilton, Ontario, Canada); Ajay Puri, MS (Ortho), Department of Surgical Oncology, Tata Memorial Centre (Mumbai, India); R Lor Randall, MD, FACS, Department of Orthopaedic Surgery, University of California, Davis (Sacramento, California, USA); Patricia Schneider, BSc, Department of Surgery, McMaster University (Hamilton, Ontario, Canada); Sheila Sprague, PhD, Department of Surgery, McMaster University (Hamilton, Ontario, Canada); Nina Szpakowski, MSc, DVM, (Community); Lehana Thabane, PhD, Department of Health Research Methods, Evidence and Impact, McMaster University (Hamilton, Ontario, Canada); Robert Turcotte, MD, FRCSC, Department of Surgery, McGill University (Montreal, Quebec, Canada); Roberto Vélez, MD, PhD, Department of Orthopaedic Surgery, Hospital Vall d'Hebron (Barcelona, Catalunya, Spain); David Wilson, MD. MSc, Department of Surgery, McMaster University (Hamilton, Ontario, Canada); Kevin Zbuk, MD, FRCPC, Department of Oncology, McMaster University (Hamilton, Ontario, Canada); Gordon Guyatt, MD, FRCPC, Department of Medicine \& Department of Health Research Methods, Evidence and Impact, McMaster University (Hamilton, Ontario, Canada).

Contributors Substantial contributions to the conception or design of the work; or the acquisition, analysis or interpretation of data for the work: Michelle Ghert;
Mohit Bhandari; Anthony Bozzo; PD Sander Dijkstra; Anthony Griffin; Robert Grimer; James Hayden; Arlene Manherz; Karim Masrouha; Paula McKay; Benjamin Miller; Naveen Parasu; Ajay Puri; R. Lor Randall; Patricia Schneider; Sheila Sprague; Nina Szpakowski; Lehana Thabane; Robert Turcotte; Roberto Vélez; David Wilson; Kevin Zbuk; Gordon GuyattDrafting the work: Michelle Ghert; Patricia Schneider; Karim Masrouh;Revising it critically for important intellectual content: Michelle Ghert; Mohit Bhandari; Anthony Bozzo; PD Sander Dijkstra; Anthony Griffin; Robert Grimer; James Hayden; Arlene Manherz; Karim Masrouha; Paula McKay; Benjamin Miller; Naveen Parasu; Ajay Puri; R. Lor Randall; Patricia Schneider; Sheila Sprague; Nina Szpakowski; Lehana Thabane; Robert Turcotte; Roberto Vélez; David Wilson; Kevin Zbuk; Gordon Guyatt; Final approval of the version to be published: Michelle Ghert; Mohit Bhandari; Anthony Bozzo; P.D. Sander Dijkstra; Anthony Griffin; Robert Grimer; James Hayden; Arlene Manherz; Karim Masrouha; Paula McKay; Benjamin Miller; Naveen Parasu; Ajay Puri; R Lor Randall; Patricia Schneider; Sheila Sprague; Nina Szpakowski; Lehana Thabane; Robert Turcotte; Roberto Vélez; David Wilson; Kevin Zbuk; Gordon Guyatt; Agreement to be accountable for all aspects of the work in ensuring that questions related to the accuracy or integrity of any part of the work are appropriately investigated and resolved: Michelle Ghert; Mohit Bhandari; Anthony Bozzo; PD Sander Dijkstra; Anthony Griffin; Robert Grimer; James Hayden; Arlene Manherz; Karim Masrouha; Paula McKay; Benjamin Miller; Naveen Parasu; Ajay Puri; R Lor Randall; Patricia Schneider; Sheila Sprague; Nina Szpakowski; Lehana Thabane; Robert Turcotte; Roberto Vélez; David Wilson; Kevin Zbuk; Gordon Guyatt.

Funding This research is supported by funding through the Hamilton Academic Health Science Organiza-tion (HAHSO) and the Canadian Cancer Society Research Institute (CCSRI) Innovation Grants.

Competing interests Dr Bhandari, Dr Ghert, Dr Randall and Dr Hayden report personal fees from consultancy and/or royalties outside the submitted work.

Patient consent for publication Not required.

Ethics approval This trial has received final ethics approval from the McMaster/ Hamilton Health Sciences Research Ethics Board on 27 August 2019 (Project number 7562)

Provenance and peer review Not commissioned; externally peer reviewed.

Open access This is an open access article distributed in accordance with the Creative Commons Attribution Non Commercial (CC BY-NC 4.0) license, which permits others to distribute, remix, adapt, build upon this work non-commercially, and license their derivative works on different terms, provided the original work is properly cited, appropriate credit is given, any changes made indicated, and the use is non-commercial. See: http://creativecommons.org/licenses/by-nc/4.0/.

\section{REFERENCES}

1. Pervaiz N, Colterjohn N, Farrokhyar F, et al. A systematic metaanalysis of randomized controlled trials of adjuvant chemotherapy for localized resectable soft-tissue sarcoma. Cancer 2008;113:573-81.

2. Whooley BP, Mooney MM, Gibbs JF, et al. Effective follow-up strategies in soft tissue sarcoma. Semin Surg Oncol 1999;17:83-7.

3. Whooley BP, Gibbs JF, Mooney MM, et al. Primary extremity sarcoma: what is the appropriate follow-up? Ann Surg Oncol 2000;7:9-14.

4. Kane JM. Surveillance strategies for patients following surgical resection of soft tissue sarcomas. Curr Opin Oncol 2004;16:328-32.

5. Gadd MA, Casper ES, Woodruff JM, et al. Development and treatment of pulmonary metastases in adult patients with extremity soft tissue sarcoma. Ann Surg 1993;218:705-12.

6. Huth JF, Eilber FR. Patterns of metastatic spread following resection of extremity soft-tissue sarcomas and strategies for treatment. Semin Surg Oncol 1988;4:20-6.

7. Songür N, Dinç M, Özdilekcan C, et al. Analysis of lung metastases in patients with primary extremity sarcoma. Sarcoma 2003;7:63-7.

8. The ESMO/European Sarcoma Network Working Group. Bone sarcomas: ESMO clinical practice guidelines for diagnosis, treatment and follow-up. Annals of Oncology 2014;25(suppl 3):iii113-23.

9. The ESMO/European Sarcoma Network Working Group. Soft tissue and visceral sarcomas: ESMO clinical practice guidelines for diagnosis, treatment and follow-up. Annals of Oncology 2014;25(suppl 3):iii102-12.

10. Gerrand $\mathrm{CH}$, Billingham LJ, Woll PJ, et al. Follow up after primary treatment of soft tissue sarcoma: a survey of current practice in the United Kingdom. Sarcoma 2007;2007:34128

11. Greenberg DD, Crawford B. Surveillance strategies for sarcoma: results of a survey of members of the musculoskeletal tumor Society. Sarcoma 2016;2016:8289509 
12. Ries Z, Gibbs CP, Scarborough MT, et al. Pulmonary surveillance strategies following sarcoma excision vary among orthopedic oncologists: a survey of the musculoskeletal tumor Society. lowa Orthop J 2016;36:109-16.

13. von Mehren M, Randall RL, Benjamin RS, et al. Soft tissue sarcoma, version 2.2018, NCCN clinical practice guidelines in oncology. J Natl Compr Cancer Netw 2018;16:536-63.

14. Bozzo AGM, Baldawi H, Simchovich G. 2018. Optimal surveillance strategies following curative surgery for extremity sarcoma: A systematic review of randomized control trials. Open Science Framework.

15. Puri A, Gulia A, Hawaldar R, et al. Does intensity of surveillance affect survival after surgery for sarcomas? results of a randomized noninferiority trial. Clin Orthop Relat Res 2014;472:1568-75.

16. Puri A, Ranganathan P, Gulia A, et al. Does a less intensive surveillance protocol affect the survival of patients after treatment of a sarcoma of the limb? updated results of the randomized toss study. Bone Joint J 2018;100-B:262-8.

17. Goel A, Christy M, Virgo K, et al. Costs of follow-up after potentially curative treatment for extremity soft-tissue sarcoma. Int $\mathrm{J}$ Oncol 2004;25:429-35.

18. Longo CJ, Deber R, Fitch M, et al. An examination of cancer patients' monthly 'out-of-pocket' costs in Ontario, Canada. Eur J Cancer Care 2007;16:500-7.

19. Hopkins RB, Goeree R, Longo CJ. Estimating the National wage loss from cancer in Canada. Curr Oncol 2010;17:40-9.

20. Nipp RD, Zullig LL, Samsa G, et al. Identifying cancer patients who alter care or lifestyle due to treatment-related financial distress. Psychooncology 2016;25:719-25.

21. Thompson CA, Charlson ME, Schenkein E, et al. Surveillance CT scans are a source of anxiety and fear of recurrence in long-term lymphoma survivors. Ann Oncol 2010;21:2262-6.
22. Thomas S, GLYNNE-JONES ROB, Chait IAN. Is it worth the wait? A survey of patients' satisfaction with an oncology outpatient clinic. Eur J Cancer Care 1997;6:50-8.

23. Brenner DJ, Hall EJ. Computed tomography - an increasing source of radiation exposure. N Engl J Med 2007;357:2277-84.

24. Schneider PJ, Evaniew N, McKay P, et al. Moving forward through consensus: a modified Delphi approach to determine the top research priorities in orthopaedic oncology. Clin Orthop Relat Res 2017;475:3044-55.

25. Freidlin B, Korn EL. Two-by-Two factorial cancer treatment trials: is sufficient attention being paid to possible interactions? J Nat/ Cancer Inst 2017;109.

26. Moore CG, Carter RE, Nietert PJ, et al. Recommendations for planning pilot studies in clinical and translational research. Clin Transl Sci 2011;4:332-7.

27. Investigators $\mathrm{P}$. Prophylactic antibiotic regimens in tumour surgery (PARITY): a pilot multicentre randomised controlled trial. Bone Joint Res 2015;4:154-62.

28. Zelle BA, Bhandari M, Sanchez Al, et al. Loss of follow-up in orthopaedic trauma: is $80 \%$ follow-up still acceptable? J Orthop Trauma 2013;27:177-81.

29. Thabane L, Ma J, Chu R, et al. A tutorial on pilot studies: the what, why and how. BMC Med Res Methodol 2010;10:1.

30. Ellis LM, Bernstein DS, Voest EE, et al. American Society of clinical oncology perspective: raising the bar for clinical trials by defining clinically meaningful outcomes. J Clin Oncol 2014;32:1277-80.

31. Leon AC, Davis LL, Kraemer HC. The role and interpretation of pilot studies in clinical research. J Psychiatr Res 2011;45:626-9.

32. Moher D, Schulz KF, Altman DG. The CONSORT statement: revised recommendations for improving the quality of reports of parallelgroup randomised trials. The Lancet 2001;357:1191-4.

33. Montgomery AA, Peters TJ, Little P. Design, analysis and presentation of factorial randomised controlled trials. BMC Med Res Methodol 2003;3:26. 